\title{
Individual heterogeneity in erythrocyte susceptibility to Babesia divergens is a critical factor for the outcome of experimental spleen-intact sheep infections
}

\author{
Laurence Malandrin $^{1 *}$, Maggy Jouglin ${ }^{1}$, Emmanuelle Moreau $^{2}$, \\ Alain CHAUvin ${ }^{2}$ \\ ${ }^{1}$ INRA, UMR 1300 BioEpAR, ENVN, Atlanpôle - La Chantrerie, BP 40706, F-44307 Nantes Cedex 03, France \\ ${ }^{2}$ École Nationale Vétérinaire, UMR 1300 BioEpAR, ENVN, Atlanpôle - La Chantrerie, BP 40706, \\ F-44307 Nantes Cedex 03, France
}

(Received 20 August 2008; accepted 25 February 2009)

\begin{abstract}
Susceptibility of sheep erythrocytes to Babesia divergens was investigated in vitro and a high inter-individual variability in their ability to support parasite population development was demonstrated, with some individuals having refractory red blood cells (RBC). As neither changes in growth conditions nor the use of different $B$. divergens strains influenced the level of susceptibility, the main factor postulated for this variability is the erythrocyte itself. Sheep therefore represent an excellent in vitro model to study the parasite-erythrocyte interaction. In addition, the existence of refractory RBC should help in the identification of the erythrocyte components required for $B$. divergens development. Experimental infections were carried out on spleen-intact sheep characterized by refractory or fully susceptible erythrocyte types. These differences translated into the successful infection of only those animals with susceptible erythrocytes: infected animals showed no clinical signs, but maintained an asymptomatic persistent infection, as usually observed in the natural bovine host. Sheep therefore represent model organisms that can allow us to study interactions between $B$. divergens and its vertebrate host at different levels of biological organisation, from the target cell to the intact animal, and represent an experimental infection model of concomitant immunity. Only a low percentage $(13 \%)$ of the sheep population tested possessed susceptible erythrocytes and the potential role of sheep as a natural host or reservoir of $B$. divergens is discussed.
\end{abstract}

Babesia divergens / sheep / erythrocyte / red blood cell / experimental animal model

\section{INTRODUCTION}

Babesia divergens, the main agent of bovine babesiosis in Europe, is an Apicomplexan parasite transmitted to its vertebrate host by the tick Ixodes ricinus. In cattle, the intra-erythrocytic asexual development of the parasite rarely causes serious disease (fever, anemia and hemoglobinuria), and asymptomatic subclinical

\footnotetext{
* Corresponding author: malandrin@vet-nantes.fr
}

infections and carriers are far more frequently encountered [28].

$B$. divergens appears to be less host specific than other Babesia species. In vitro, continuous culture has been described for rat, bovine, ovine and human erythrocytes and short-term survival in horse or donkey red blood cells (RBC) is reported $[21,26]$. The experimental infection of splenectomized primate, rat, sheep, mouflon, red, roe and fallow deer has been achieved, as well as of non-splenectomized reindeer and

This is an Open Access article distributed under the terms of the Creative Commons Attribution-Noncommercial License (http://creativecommons.org/licenses/by-nc/3.0/), which permits unrestricted use, distribution, and reproduction in any noncommercial medium, provided the original work is properly cited. 
cattle [28]. The Mongolian gerbil (Meriones unguiculatus) is the main, fully susceptible laboratory experimental model used to study $B$. divergens infections [15]. However, in contrast to the natural bovine host, it develops an acute and often fatal babesiosis, and, if it recovers, shows sterile rather than concomitant immunity [28]. Our aim is therefore to develop an in vitro model to study the molecular interactions of $B$. divergens with its target cell, the erythrocyte and thus to understand the biochemical basis of susceptibility, with the possibility to analyse the infection process on animals with erythrocytes of different characteristics. Several features led us to select sheep as a possible model. First, $B$. divergens can be cultivated in vitro in sheep erythrocytes and splenectomized sheep can be successfully infected and can maintain lowparasitemia infections [5]. Furthermore, discrepancies of experimental infection outcomes in sheep between $[5,7]$ indicated possible differences in susceptibility among animals. In a study on the erythrocyte invasion of B. bovis, the greatest differences in invasion efficiencies within a species were also obtained with ovine erythrocytes [9]. These observations led us to analyse the susceptibility of sheep erythrocytes to $B$. divergens by comparing the abilities of $\mathrm{RBC}$ from different individual animals to sustain $B$. divergens population growth in vitro. The term susceptibility used in this paper refers to the ability of the erythrocytes to support parasite population growth, with no distinction among the ability of the parasite to penetrate into the $\mathrm{RBC}$, to develop intracellularly, or to egress. The influence of growth conditions and parasite isolates on culture population growth in different erythrocytes were also investigated. Sheep with either fully susceptible RBC or refractory $\mathrm{RBC}$ were then selected to perform experimental infections of intact animals and to compare infection outcomes.

\section{MATERIALS AND METHODS}

\subsection{Origins of animal erythrocytes}

The 19 sheep used throughout this study included two sheep of the breed "Belle Islois" (918 and 919) as well as 17 sheep of the breed "Vendéen". Another population of 30 "Vendéen" sheep was also randomly tested for the susceptibility of their RBC to $B$. divergens in vitro. Peripheral blood was collected as previously described $[17,18]$.

\subsection{B. divergens isolates, in vitro culture and population growth}

To evaluate the influence of the $B$. divergens strain on the ability to grow in different erythrocytes, we selected 12 isolates of human (Rouen 87) or bovine origin from different geographical regions of France. The cattle isolates included strains were obtained from both the acute $(0904 \mathrm{~B}, 2802 \mathrm{C}$ and 3509B) and the carrier stage $(7153,7656, \mathrm{C} 244$, C246, C597, IV29, IV101 and IV135). One clonal line was selected for each isolate from dilution limit experiments where clonality was confirmed by less than $20 \%$ positive wells. Molecular variability of these 12 clonal lines was assessed by PCR-RFLP on $b d 37$ gene (data not shown) [12].

$B$. divergens was cultivated in sheep erythrocytes with RPMI 1640 supplemented with 10\% Fetal Calf Serum (FCS, Cambrex, Verviers, Belgium) [5, 17]. In all experiments, a growth reference was included (sheep 919 erythrocytes). This donor animal was selected on the basis of a high in vitro growth rate and was used to maintain $B$. divergens cultures in routine.

Growth was monitored by measuring parasitemia in a minimum of $2000 \mathrm{RBC}$. When parasites could not be detected in the first $2000 \mathrm{RBC}$, up to 10000 $\mathrm{RBC}$ were examined. Parasitemia was determined for each individual culture RBC pellet or for the pooled infected Red Blood Cell (iRBC) from triplicate cultures.

As spectrophotometric hemoglobin measurement in the culture supernatant was demonstrated to be a less labor-intensive and more precise method to monitor $B$. divergens growth in vitro [18], we decided to use it for our purpose in part of our experiments. This method is based on hemoglobin release measured after parasite multiplication and RBC lysis. However, it could be biased by the variability in Mean Corpuscular Hemoglobin from one sheep to another. The correlation between parasitemia and $\mathrm{A}_{405}$ was therefore verified for the erythrocytes of the 19 sheep used and for two clones, Rouen F5 and 2802C D5, before further use of this method. $\mathrm{A}_{405}$ was measured as previously described, and for each in vitro condition and individual tested, a parasite-free control culture was included in triplicate to measure erythrocyte self lysis, the mean absorbance of this control was 
then substracted from the corresponding test value [18]. Culture conditions are detailed in Table I.

\subsection{In vitro susceptibility of sheep erythrocytes}

To test the susceptibility of sheep erythrocytes to $B$. divergens, we compared parasite population growth rates. The susceptibility measurements of the 19 sheep were performed in four separate experiments (conditions described in Tab. I). For each experiment, sampling time and growth measurement method, a rank was attributed to each sheep according to the ability of its erythrocytes to sustain $B$. divergens growth. Rank 1 was attributed to the sheep with highly susceptible erythrocytes while rank 19 was attributed to the sheep with refractory erythrocytes (Tab. I). For each animal, the rankings obtained across experiments (11 values/sheep) were averaged and the values obtained were used to classify the sheep according to the susceptibility of their erythrocytes.

\subsection{Influence of growth medium on sheep erythrocyte susceptibility}

As in vitro growth is reported to be affected by the medium composition [29], the effect of sera type on susceptibility and growth was tested in two clones of $B$. divergens (Rouen F5 or $2802 \mathrm{C}$ D5) using erythrocytes prepared from five sheep. Five different FCS (from three manufacturers: Cambrex, Eurobio - Les Ulis, France - and Sigma - St-Quentin-Fallavier, France) and two different sheep sera (Cambrex, Eurobio) were compared. Both $B$. divergens clones were first cultivated with each type of sera before their inoculation at $4.10^{5} \mathrm{iRBC} / \mathrm{mL}$ in the test medium. The effect of sera concentration $(10,20,30$ and $40 \%$ ) was tested for one sheep and one calf sera on a selection of 4 sheep erythrocyte types using the same $B$. divergens clones (Rouen F5 or 2802C D5) and an inoculation concentration of $2.10^{5} \mathrm{iRBC} / \mathrm{mL}$.

\subsection{Variation in susceptibility according to $B$. divergens strain}

Eight sheep were selected based on differences in the susceptibility of their erythrocytes and were tested against the $12 \mathrm{~B}$. divergens clonal lines. Cultures were performed with standardized growth conditions $\left(1.10^{9} \mathrm{RBC} / \mathrm{mL}, 10 \%\right.$ sheep serum, 96 -well microtiter plates, triplicates), and inoculated with equal $\left(2.10^{5} \mathrm{iRBC} / \mathrm{mL}\right.$, one experiment) or varying amounts of parasites according to their growth rates (two experiments). The results presented correspond to the third experiment where the parasite inoculation concentration and the sampling time were adjusted according to the growth rate of each parasite clonal line. These features were evaluated from the two previous experiments (data not shown).

\subsection{Evaluation of prevalence of susceptible sheep in a random population}

Blood was collected from 30 "Vendéen" sheep and the culture medium was inoculated at $4.10^{5}$ $\mathrm{iRBC} / \mathrm{mL}$ with two $B$. divergens clones (Rouen F5 and $2802 \mathrm{C} \mathrm{D5}$ ) in the presence of $10 \%$ sheep serum. This experiment was repeated twice on the same sheep population.

\subsection{Experimental infection of non-splenectomized sheep}

Six sheep were selected from the previous experiments: four with susceptible erythrocytes (3198, 3464, 3489 and 3490) and two with non-susceptible erythrocytes (8 and 28). They were all infected with $B$. divergens Rouen 1987 reisolated from an experimentally infected splenectomized sheep [5] and preserved in liquid nitrogen. To infect susceptible sheep, the isolate was cultivated with each of the four susceptible erythrocytes collected 3 weeks before infection. To infect sheep with non-susceptible RBC, erythrocytes from our donor (919) were used. Two sheep were infected with their own PBS washed iRBC $\left(1.10^{10}\right.$ iRBC) either intra-venously (sheep 3464) or intra-peritoneally (sheep 3490). For the four remaining infections, free merozoites were isolated by centrifugation ( $15 \mathrm{~min}, 2500 \mathrm{~g}$ ) on a Percoll solution adjusted to a density of $1.08 \mathrm{~g} / \mathrm{mL}$, and were washed with PBS (15 min, $2500 \mathrm{~g}$ ) before use. Presence, viability and infectivity of the collected merozoites were verified respectively by the microscopic examination of Giemsa-stained smears, the in vitro culture and the inoculation of gerbils (M. unguiculatus). The approximate amount of viable injected merozoites was determined in vitro by the limiting dilution technic using a ten-fold dilution series. Merozoites (about $5.10^{6}$ ) were injected either intra-peritoneally or sub-cutaneously.

The establishment and maintenance of the parasite in the bloodstream was monitored by in vitro amplification of the parasite from blood collected at weeks $1,3,6$ and 8 post-infection as described previously [17]. In the case of sheep with non-susceptible erythrocytes, cultures were attempted 18 times over a period of 3 weeks. The sheep humoral response to 
Table I. Erythrocyte susceptibility of the 19 tested sheep estimated by the growth of $B$. divergens Rouen 87 F5. For each experiment and each sampling time, sheep were attributed a number (from 1 to 19) corresponding to the susceptibility of their erythrocytes (from the most - number 1 - to the least - number 19 - susceptible). Sheep were then classified according to the mean rank obtained across experiments. The separation of sheep into three groups was performed by the dynamic clustering method (Xlstat) on these susceptibility rankings. The table summarizes the classification based on 4 separate experiments where susceptibility was estimated at different time points by parasitemia counts ( $\mathrm{P}$ in bold) and/or $\mathrm{A}_{405}$ measurements of the corresponding culture supernatant (A). Parasitemia data are either the mean of two repetitions (Exp. 1) or the parasitemia of the iRBC pellet obtained by mixing erythrocytes from the three cultures used to measure the absorbance (Exp. 2 and 3 ). Absorbance data used are the mean of triplicate cultures.

\begin{tabular}{|c|c|c|c|c|c|c|c|c|c|c|c|c|}
\hline Experimental conditions & \multicolumn{2}{|c|}{ Exp. 1} & \multicolumn{4}{|c|}{ Exp. 2} & \multicolumn{3}{|c|}{ Exp. 3} & \multicolumn{2}{|c|}{ Exp. 4} & \\
\hline $\begin{array}{l}\text { Inoculation dose } \\
(\mathrm{iRBC} / \mathrm{mL})\end{array}$ & \multicolumn{2}{|c|}{$2.10^{5}$} & \multicolumn{4}{|c|}{$2.10^{5}$} & \multicolumn{3}{|c|}{$2.10^{6}$} & \multicolumn{2}{|c|}{$2.10^{5}$} & \\
\hline Plate format & \multirow{2}{*}{\multicolumn{2}{|c|}{$\begin{array}{c}24 \text { wells } \\
68,88\end{array}$}} & \multirow{2}{*}{\multicolumn{4}{|c|}{$\begin{array}{c}96 \text { wells } \\
72,89\end{array}$}} & \multirow{2}{*}{\multicolumn{3}{|c|}{$\begin{array}{c}96 \text { wells } \\
49,66\end{array}$}} & \multirow{2}{*}{\multicolumn{2}{|c|}{$\begin{array}{c}96 \text { wells } \\
72,89\end{array}$}} & \\
\hline Sampling times (h) and & & & & & & & & & & & & \\
\hline method $(\mathbf{P}$ or $\mathrm{A})$ & \multicolumn{2}{|c|}{$\mathbf{P}$} & \multicolumn{4}{|c|}{$\mathbf{P}, \mathrm{A}$} & \multicolumn{3}{|c|}{$\mathbf{P}, \mathrm{A}$} & \multicolumn{2}{|c|}{ A } & \\
\hline & $68 \mathrm{P}$ & 88P & $72 P$ & $72 \mathrm{~A}$ & $89 P$ & $89 \mathrm{~A}$ & $49 P$ & $49 \mathrm{~A}$ & $66 \mathrm{~A}$ & $72 \mathrm{~A}$ & $89 \mathrm{~A}$ & \\
\hline Sheep reference & & & & & & & & & & & & $\begin{array}{l}\text { Mean } \\
\text { ranking }\end{array}$ \\
\hline \multicolumn{13}{|l|}{ Highly susceptible } \\
\hline 919 & 1 & 1 & 1 & 1 & 2 & 1 & 3 & 1 & 1 & 1 & 1 & 1.3 \\
\hline 3490 & 5 & 3 & 2 & 2 & 1 & 2 & 2 & 4 & 3 & 2 & 2 & 2.6 \\
\hline 918 & 3 & 5 & 3 & 6 & 3 & 3 & 1 & 3 & 4 & 4 & 4 & 3.6 \\
\hline 3264 & 2 & 2 & 9 & 8 & 6 & 8 & 5 & 2 & 2 & 3 & 3 & 4.6 \\
\hline 3464 & 7 & 6 & 5 & 3 & 5 & 4 & 4 & 5 & 5 & 7 & 7 & 5.3 \\
\hline 3342 & 8 & 9 & 4 & 4 & 4 & 6 & 8 & 7 & 6 & 6 & 5 & 6.1 \\
\hline 3489 & 9 & 7 & 6 & 9 & 8 & 9 & 6 & 6 & 7 & 5 & 6 & 7.1 \\
\hline 3198 & 6 & 4 & 8 & 5 & 7 & 5 & 9 & 9 & 9 & 9 & 9 & 7.3 \\
\hline 3466 & 4 & 8 & 7 & 7 & 9 & 7 & 7 & 8 & 8 & 8 & 8 & 7.6 \\
\hline \multicolumn{13}{|l|}{ Moderately susceptible } \\
\hline 3390 & 10 & 14 & 10 & 12 & 11 & 12 & 10 & 10 & 10 & 10 & 10 & 10.8 \\
\hline 3442 & 12 & 13 & 11 & 10 & 10 & 11 & 12 & 14 & 13 & 13 & 14 & 12.1 \\
\hline 3295 & 14 & 10 & 14 & 11 & 14 & 10 & 13 & 11 & 12 & 17 & 13 & 12.6 \\
\hline 3463 & 11 & 15 & 17 & 15 & 16 & 17 & 11 & 13 & 11 & 14 & 12 & 13.8 \\
\hline 3446 & 13 & 12 & 15 & 13 & 15 & 14 & 17 & 16 & 16 & 11 & 11 & 13.9 \\
\hline 3533 & 15 & 16 & 13 & 16 & 12 & 15 & 14 & 12 & 14 & 12 & 15 & 14.0 \\
\hline 3454 & 17 & 11 & 12 & 14 & 13 & 16 & 16 & 17 & 17 & 16 & 17 & 15.1 \\
\hline 3448 & 16 & 17 & 16 & 17 & 17 & 13 & 15 & 15 & 15 & 15 & 16 & 15.6 \\
\hline \multicolumn{13}{|l|}{ Non susceptible } \\
\hline 3476 & 18 & 18 & 18 & 18 & 18 & 18 & 18 & 18 & 18 & 18 & 18 & 18.0 \\
\hline 3477 & 19 & 19 & 19 & 19 & 19 & 19 & 19 & 19 & 19 & 19 & 19 & 19.0 \\
\hline
\end{tabular}

parasite presence and/or subsequent potential multiplication was monitored by ELISA. Free merozoites, prepared as described above, were used as antigens.
They were mechanically lysed by freeze-thawing, treated with DNAse $(0.1 \mathrm{mg} / \mathrm{mL}$ for $1 \mathrm{~h}$ at room temperature) and $\operatorname{SDS}(0.3 \%$ final concentration) that 


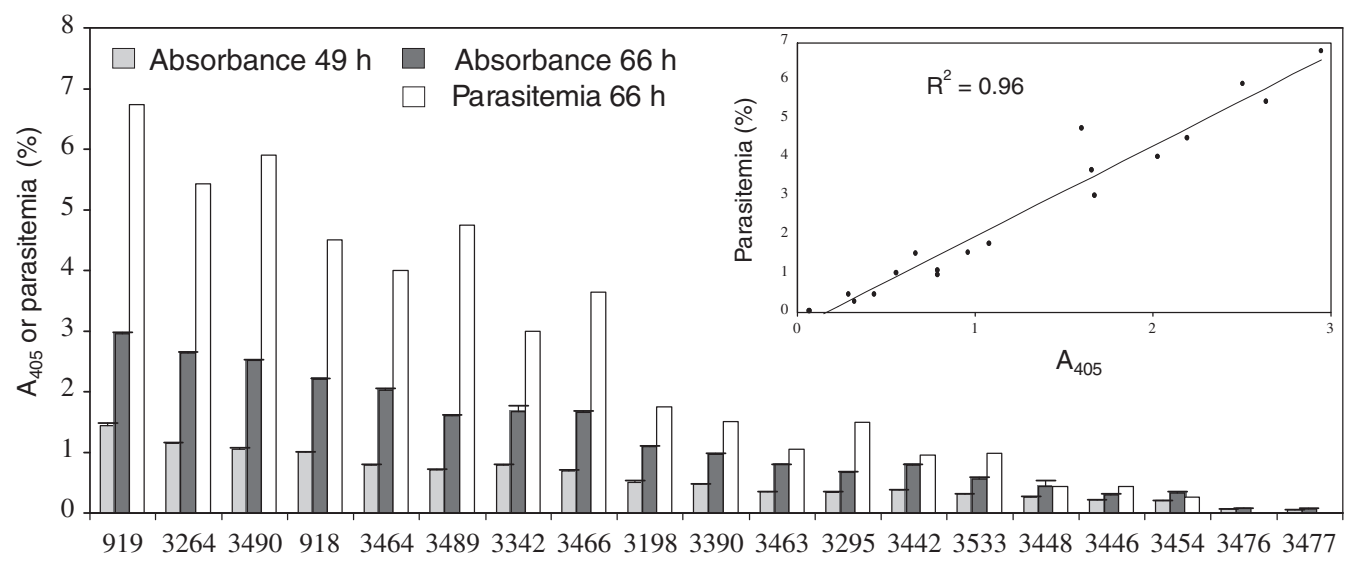

Sheep reference number

Figure 1. Variability in the susceptibility of erythrocytes prepared from 19 sheep. $\mathrm{A}_{405}$ of the culture supernatants and counts of parasitemia were measured 49 and $66 \mathrm{~h}$ after culture inoculation with B. divergens clone $2802 \mathrm{C}$ D5 $\left(2.10^{6} \mathrm{iRBC} / \mathrm{mL}\right)$ in $2 \mathrm{~mL}$-well (RPMI 1640 , sheep serum at $10 \%, 1.10^{9}$ $\mathrm{RBC} / \mathrm{mL}$ ) for each sheep. Each $\mathrm{A}_{405}$ data point represents the average value of the triplicate culture pellets, and the error bars indicate the standard deviation. Parasitemia was calculated from the RBC obtained by mixing the triplicate culture pellets. In the insert, the regression analysis performed on the data from each method at $66 \mathrm{~h}$ post-inoculation is shown, as well as the regression coefficient. This experiment was also performed with the clone Rouen F5 with similar results (see Tab. I; Exp. 3).

was subsequently eliminated with Amicon centrifugal filter devices (Millipore, Billerica, USA). The ELISA was performed with an anti-IgG conjugate as described previously except for the antigen used (plates were coated with $2.5 \mu \mathrm{g} / \mathrm{mL}$ of the prepared antigen) [25].

\subsection{Statistical analysis}

Results shown are means \pm standard deviations of triplicate cultures from the representative experiments. Each experiment was repeated at least twice or performed in parallel with two clones. The dynamic clustering method (Xlstat) was used to distinguish different susceptibility groups in the tested sheep.

\section{RESULTS}

\subsection{In vitro heterogeneity of sheep erythrocyte susceptibility to $B$. divergens}

Extreme differences among individuals were observed in the ability of their erythrocytes to sustain $B$. divergens $2802 \mathrm{C}$ D5 growth in vitro (Fig. 1), ranging from $87.5 \%$ (sheep $3490)$ to $0 \%$ (3477) relative to the parasitemia of the control erythrocytes (919). Giemsastained smears performed after 7 days in culture showed no development in sheep erythrocytes 3476 and 3477.

Direct counts of parasitemia, as well as $\mathrm{A}_{405}$ measurements, revealed this heterogeneity in susceptibility and a good correlation $(r=0.96)$ was found between methods (Fig. 1, insert). The reproductibility of this heterogeneity was tested by conducting four separate experiments with the clone Rouen F5 on the same 19 animals. Regardless of the experimental conditions used, erythrocyte susceptibility did not vary extensively from one experiment to another: the erythrocytes of the donor always allowed the best growth and sheep 3476 and 3477 always showed non-susceptible erythrocytes. Three groups were distinguished using the dynamic clustering method on the mean ranking: sheep with highly (1-9), moderately (10-17) and refractory RBC (18 and 19) 

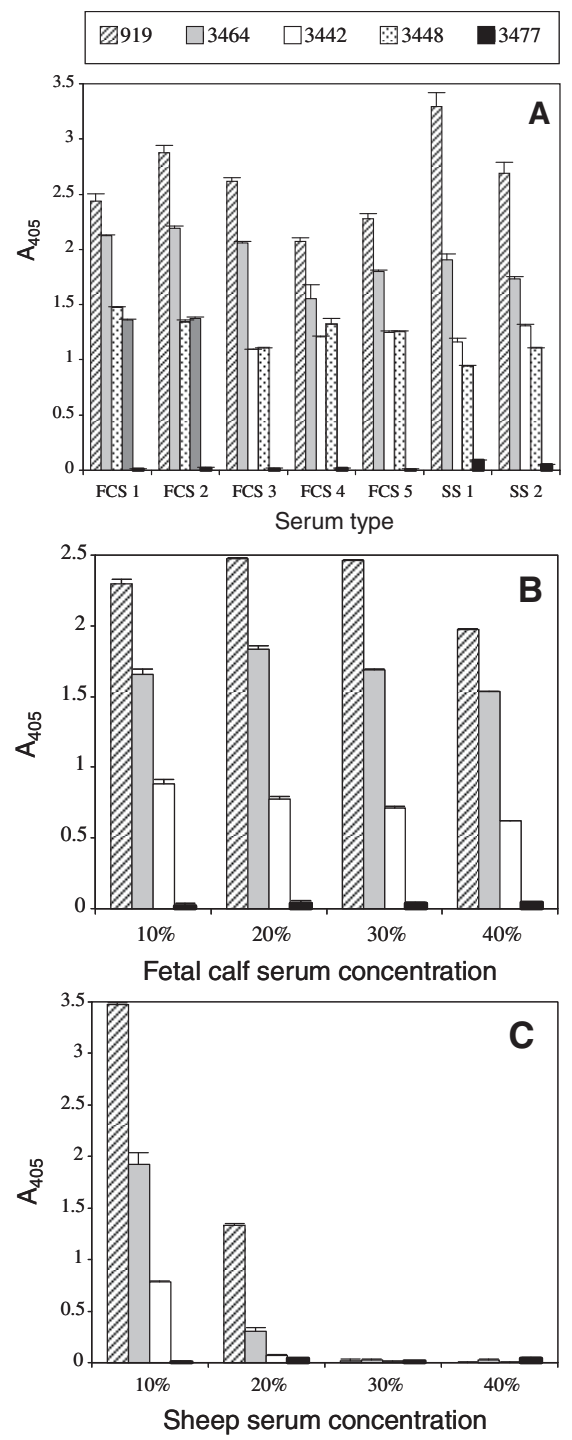

Figure 2. Influence of medium composition on the observed variability of erythrocyte susceptibility. $B$. divergens clone $2802 \mathrm{C}$ D5 was inoculated into media prepared with RBC of varying degrees of susceptibility and sera from different origins (5 different FCS - and 2 different sheep sera - SS) used at $10 \%(\mathrm{~A})$ or at different concentrations (10, 20, 30 and $40 \%$ ) (B and C). Each $\mathrm{A}_{405}$ data point represents the average value of the triplicate wells, and the error bars indicate the standard deviation. This experiment was also conducted with the clone Rouen F5 with similar results.
(Tab. I). This separation was performed only to enable the selection of representatives of each group for further experiments.

\subsection{Influence of growth medium on sheep erythrocytes susceptibility}

Whatever the type, the commercial source (Fig. 2A), or the concentration of serum used (Figs. 2B and 2C), the distribution of sheep erythrocyte susceptibility remained unchanged. Suitability of different FCS in supporting $B$. divergens growth varied only slightly: from 85 to $117 \%$ of the control $(919$, FCS1). Sheep serum at a concentration of $20 \%$ or above was clearly detrimental to parasite development. Thus, overall the modification or enrichment of the growth medium tested did not permit $B$. divergens to develop in non-susceptible erythrocytes.

\subsection{In vitro variability of sheep erythrocyte susceptibility according to $B$. divergens clonal lines}

Erythrocyte susceptibility from 8 sheep to a selection of $12 \mathrm{~B}$. divergens clones was compared and presented in Figure 3. The same susceptibility gradient was conserved independently of the strain used. Slight differences were noticed in the case of slow-growing strains (7656 and C246) with moderately susceptible erythrocytes (3448). All clones grew well in highly susceptible sheep erythrocytes, except 0904B which appeared particularly well adapted to the donor erythrocytes (919). The non-susceptible erythrocytes from sheep 3477 did not sustain the growth of any $B$. divergens clones, even if a low $\mathrm{A}_{405}$ could be observed in certain cases (i.e. 7656) (stained smears were evaluated at the end of the experiment and a parasitemia $<0.1 \%$ was found corresponding to the inoculated parasites).

\subsection{Evaluation of prevalence of susceptible sheep in a random population}

The prevalence of sheep with susceptible erythrocytes was analyzed in a small population 


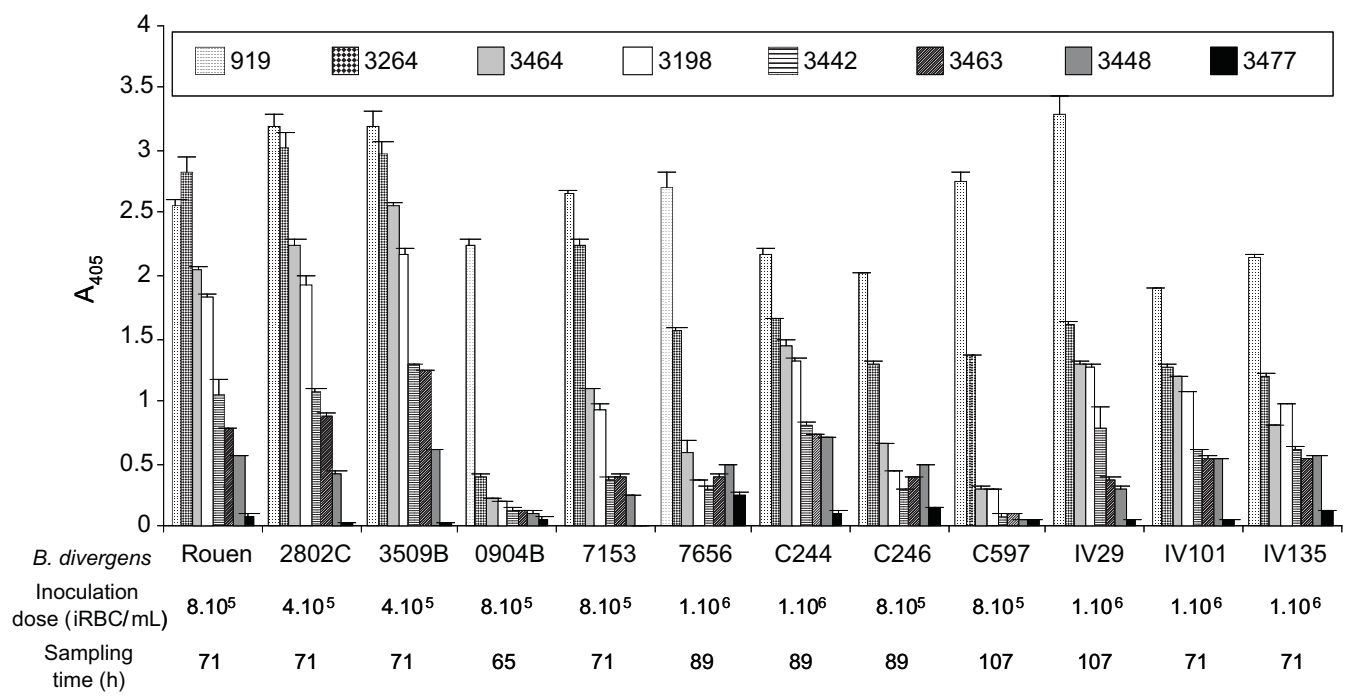

Figure 3. Influence of $B$. divergens clone on the observed variability of erythrocyte susceptibility. The susceptibility of the erythrocytes from 8 sheep to clones obtained from different $B$. divergens isolates was tested. Each $\mathrm{A}_{405}$ data point represents the average value of the triplicate wells, and the error bar indicates the standard deviation. This experiment was performed three times: once with the inoculation of the clones at the same concentration and twice with inoculation concentrations adapted to the growth rate of each clone (only results from the last run are presented here). Similar results were obtained from these experiments.

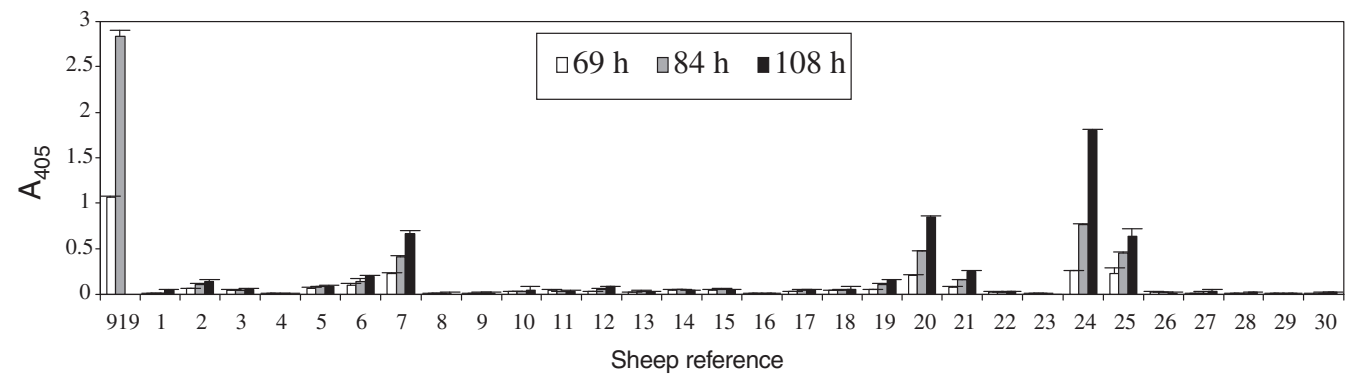

Figure 4. Evaluation of the prevalence of susceptible sheep in a random population. The susceptibility of the erythrocytes of 30 sheep ("Vendéen" breed) was tested in vitro with B. divergens clone Rouen F5. Each $\mathrm{A}_{405}$ data point represents the average value of triplicate measures, and the error bar indicates the standard deviation. This experiment was performed twice with similar results.

of 30 sheep from the same breed ("Vendéen") with the clonal line Rouen F5 (Fig. 4). The proportion of sheep with susceptible RBC was found to be low (13\%, sheep references 7, 20, 24 and 25 on Fig. 4) and most of them possessed non-susceptible erythrocytes $(70 \%)$.

\subsection{Experimental infections of spleen-intact sheep}

Regardless of the infection route (intravenous, intraperitoneal or subcutaneous) or the parasite form (iRBC or merozoites), parasites 
Table II. Humoral response of sheep to infection by B. divergens. Spleen-intact sheep with in vitro refractory (R) or susceptible (S) erythrocytes were infected with $B$. divergens Rouen F5. At 5 time points, the humoral response was monitored by ELISA (raw values corresponding to the mean absorbance of triplicate wells and standard deviations are indicated).

\begin{tabular}{lcccccr}
\hline & \multicolumn{5}{c}{ Sheep reference (parasite form and infection route) } \\
\cline { 2 - 7 } & $8^{\mathrm{a}} \mathrm{R}(\mathrm{M}-\mathrm{IP})$ & $28^{\mathrm{a}} \mathrm{R}(\mathrm{M}-\mathrm{SC})$ & $3464 \mathrm{~S}$ (iRBC $-\mathrm{IV})$ & $3490 \mathrm{~S}(\mathrm{iRBC}-\mathrm{IP})$ & $3198 \mathrm{~S}(\mathrm{M}-\mathrm{IP})$ & $3489 \mathrm{~S}(\mathrm{M}-\mathrm{SC})$ \\
\hline Day of infection & $0.128 \pm 0.006$ & $0.127 \pm 0.008$ & $0.173 \pm 0.012$ & $0.088 \pm 0.04$ & $0.059 \pm 0.016$ & $0.101 \pm 0.026$ \\
Week 1 post infection & $0.171 \pm 0.014$ & $0.162 \pm 0.021$ & $0.213 \pm 0.009$ & $0.289 \pm 0.013$ & $0.242 \pm 0.029$ & $0.29 \pm 0.039$ \\
Week 3 post infection & $0.322 \pm 0.025$ & $0.417 \pm 0.036$ & $0.612 \pm 0.021$ & $0.737 \pm 0.068$ & $0.589 \pm 0.022$ & $0.6 \pm 0.021$ \\
Week 6 post infection & $0.095 \pm 0.032$ & $0.244 \pm 0.013$ & $0.783 \pm 0.004$ & $2.116 \pm 0.117$ & $0.443 \pm 0.045$ & $1.606 \pm 0.066$ \\
Week 8 post infection & nd & nd & $1.054 \pm 0.058$ & $2.168 \pm 0.085$ & $0.611 \pm 0.016$ & $1.435 \pm 0.084$ \\
\hline
\end{tabular}

${ }^{a}$ Sheep with refractory erythrocytes were selected from the sheep population described in Figure 4. Sheep were infected either with merozoites (M), or iRBC, and by intravenous (IV), intraperitoneal (IP) or subcutaneous (SC) routes. nd: Not done at that time point. 
were isolated regularly from the bloodstream of sheep with susceptible erythrocytes over a period of two months post-injection, indicating the successful infection of non-splenectomized sheep by $B$. divergens (Tab. II). Infected sheep never showed clinical signs and suffered only very mild infections with persistently low parasitemia. On the contrary, $B$. divergens was never isolated from the two sheep with non-susceptible erythrocytes (in vitro cultures attempted at 18 different days over the first 3 weeks postinfection). The ELISA of circulating antibodies $(\mathrm{IgG})$ directed against $B$. divergens indicated a strong humoral response from sheep with susceptible erythrocytes (Tab. II). A humoral response was also noticeable in sheep with non-susceptible erythrocytes, but its amplitude was far lower and its level decreased between the third and the sixth week post-infection. These features correspond to the sheep's response to the establishment and multiplication of the parasite in the case of animals with susceptible $\mathrm{RBC}$, and to the humoral response against the injected non-developing parasites in the case of sheep with refractory erythrocytes.

\section{DISCUSSION}

In the present study, we analysed sheep erythrocyte susceptibility to $B$. divergens and achieved the first infection of intact sheep with not only iRBC, but also purified merozoites. Infection was achieved by different infection routes, including subcutaneous, a route closer to the natural parasite transmission pathway.

$B$. divergens showed considerable variation in growth according to the sheep erythrocyte origin. This heterogeneity in susceptibility was found not only within the species Ovis aries (sheep), but also among individuals within the same sheep breed (breed "Vendéen"). The reproductibility of this heterogeneity was strong from one experiment to another. Absolute growth levels varied among experiments; this was probably due to the different experimental conditions used and the varying latency periods that depended on the status of the preculture used (exponential or not).
Significant variation among individuals of the same species in the RBC invasion efficiency by $B$. bovis was also noticed by Gaffar et al. [9] with equine and ovine erythrocytes. The greatest extent of variation was recorded with ovine $\mathrm{RBC}$, with relative invasion efficiencies to the bovine control of 30-120\%. This heterogeneity was observed solely at the invasion step since no further intraerythrocytic development followed. In our experiments, variability in parasite growth could be due to variation in one or several steps of the asexual development of the parasite $[2,20]$, i.e. the invasion, the intracellular development or the egress of merozoites from the RBC. Further experiments using methods able to separately analyse these three main steps are currently in progress to determine which step is responsible for low (or absence of) development of $B$. divergens in some erythrocytes.

Changes in the growth conditions (serum type or concentration) did not modify the heterogeneity in parasite growth rates. Therefore, the absence or slow growth of $B$. divergens in some erythrocyte types could not be explained by the presence of deleterious factors, or by the lack of necessary components in the serum used, possibilities raised by Zintl et al. [29]. As intimate molecular interactions occur with their host cell during the invasion and development of Apicomplexan parasites [11], changes in the strain used could modify the interaction between a ligand and a particular $\mathrm{RBC}$ receptor. This aspect was addressed by comparing the susceptibility of 8 sheep erythrocyte types with 12 clonal lines of the parasite. A particularly efficient combination between one clone and one erythrocyte type, resulting in a burst of growth, was not observed.

As neither variable growth conditions nor different $B$. divergens clonal lines altered the heterogeneity profile of the tested erythrocytes, it can be postulated that the main factor determining the variability in susceptibility is the erythrocyte itself. Several molecules have been identified on the surface of the RBC that act as receptors to the malarial parasite, Plasmodium sp. [10]. Knowledge on bovine Babesia are far more sparse $[14,24]$ and those on $B$. divergens rely on two recent studies, one involving 
enzymatic modification of the erythrocyte surface using human, ovine, equine and bovine RBC [27], and the other using mutant erythrocytes of human origin [16]. Both studies indicate the existence of an invasion pathway that is sialic acid-dependent, mainly via glycophorins $\mathrm{A}$ and $\mathrm{B}$. However, residual invasion suggests that alternative, sialic acid-independent pathways may also exist as already identified in the case of malaria [19]. The differences in susceptibility among erythrocyte types found in the present study could be explained by the use of invasion pathways with variable efficiency according to the receptors present on the surface of the RBC, or by variable amounts and types of sialic acid residues present on the surface of the RBC, or by the type of acid sialic linkage ( $\alpha 2-3$ or $\alpha 2-6$ ) to the underlying galactose residues $[8,9,24]$. Variations in the level and relative types of sialic acid residues have been observed among human, bovine, ovine and equine erythrocytes $[8,13]$, but there are no detailed studies on intraspecific variation. Variability in susceptibility could also be determined by factors in the erythrocyte cytoplasm which affect the parasite once it has gained access into the cell. The major cytoplasmic component is the hemoglobin, and the effects of human hemoglobin variants $(\mathrm{HbC}, \mathrm{HbE}$, $\mathrm{HbS}$ and thalassaemias) on malaria protection and in vitro growth of Plasmodium are now well documented and always involves abnormal hemoglobin types $[11,23]$. Enzymatic deficiencies such as glucose-6-phosphate dehydrogenase (G6PD) and pyruvate kinase (PK) also influence the outcome of the malaria $[11,23]$. Research on babesiosis and RBC variants is not so advanced, and the only study where typing of normal bovine hemoglobin was performed failed to show any correlation between a particular hemoglobin type and the innate resistance to babesiosis [1]. A similar analysis of sheep hemoglobins will be performed to determine if a link exists between one of the four ovine hemoglobin alleles $[3,22]$ and the susceptibility of sheep erythrocytes to $B$. divergens. The in vitro interactions between $B$. divergens and sheep erythrocytes of varying susceptibilities (fully susceptible to refractory) offers a model to perform quantitative analyses of receptors and intracellular erythrocyte components necessary to $B$. divergens development within erythrocytes.

We demonstrate here that the success of experimental infections of sheep with $B$. divergens clearly relies on the susceptibility of their erythrocytes to the parasite. This observation could explain the discrepancies between outcomes of experimental infections described in the literature $[5,7]$. We are applying the same approach to bovine erythrocytes to investigate whether such heterogeneity in erythrocyte susceptibilities could explain variation in natural susceptibility and the low clinical incidence of $B$. divergens babesiosis in cattle. We report here the first successful infection of intact sheep. The spleen is a critical effector lymphoid organ whose central role is the generation of immunity, the production of new RBC and the selective microfiltration of old, structurally modified or parasitized RBC via macrophages and monocytes [6]. Its role is essential in diseases caused by the intraerythrocytic development of parasites such as babesiosis [4] and splenectomized individuals (humans, primates, young calves, deer, rats) may become susceptible to the infection by $B$. divergens [28]. The infection of intact sheep, even when successful, did not lead to an acute form of the disease, but rather to a persistent infection of otherwise clinically healthy animals. These animals still developed a strong humoral response, thus sharing similarities with the course of infection observed in cattle. The sheep model could therefore be useful for studying the mechanisms of concomitant immunity and parasite persistence, aspects that can not be studied with gerbils and their described sterilizing immunity [28].

The sheep infection described here was performed with iRBC, but also with isolated merozoites, via the subcutaneous route as well as the intravenous and intraperitoneally ones, and with infective doses as low as $10^{6}$ merozoites. These infection conditions are thus moving closer to the natural infection route: free sporozoites injected subcutaneously via a tick bite.

The successful infection of intact sheep also raises the possibility of sheep serving as natural hosts or reservoirs for $B$. divergens. To determine the role of sheep as a potential reservoir 
for $B$. divergens, serological surveys followed by in vitro isolation and characterization of Babesia from sheep should be performed.

In conclusion, the in vitro sheep model offers a wide variety of susceptibility to $B$. divergens, including completely refractory erythrocyte types. Exploring differences in erythrocyte properties among individuals offers the opportunity for a more detailed characterisation of erythrocyte receptors and cytoplasmic components employed for the invasion and development of $B$. divergens. The sheep humoral response in this model of concomitant immunity is currently being characterized.

Acknowledgements. We wish to thank E. Lemarié, and his patience with the Dialux 20, G. Vince, $\mathrm{M}$. Billaud and $\mathrm{N}$. Brisseau for isolating $B$. divergens from asymptomatic cattle, and C. Becker for the blood collection. We are grateful to Chantal Thorin for statistical advice, Christine Kessler and Karen McCoy for the English corrections of the manuscript. Thanks are also due to the working group TMT of the REID. This project was supported by research funds from the French National Institute for Agricultural Research (INRA) and the École Nationale Vétérinaire de Nantes.

\section{REFERENCES}

[1] Bachmann A.W., Campbell R.S.F., Johnston L.A.Y., Yellowlees D., Bovine haemoglobin types and their possible relationship to resistance to babesiosis: an experimental study, Tropenmed. Parasitol. (1977) 28:361-366.

[2] Bannister L., Mitchell G., The ins, outs and roundabouts of malaria, Trends Parasitol. (2003) 19:209-213.

[3] Beale D., Lehmann H., Drury A., Tucker E.M., Haemoglobins of sheep, Nature (1966) 209: 1099-1102.

[4] Brown W.C., Norimine J., Knowles D.P., Goff W.L., Immune control of Babesia bovis infection, Vet. Parasitol. (2006) 138:75-87.

[5] Chauvin A., Valentin A., Malandrin L., L'Hostis M., Sheep as a new experimental host for Babesia divergens, Vet. Res. (2002) 33:429-433.

[6] Engwerda C.R., Beattie L., Amante F.H., The importance of the spleen in malaria, Trends Parasitol. (2005) 21:356-357.
[7] Enigk K., Friedhoff K., On the host specificity of Babesia divergens (Piroplasmidea), Z. Parasitenk. (1962) 21:238-256 (in German).

[8] Eylar E.H., Madoff M.A., Brody O.V., Oncley J.L., The contribution of sialic acid to the surface charge of the erythrocyte, J. Biol. Chem. (1962) 237:1992-2000.

[9] Gaffar F.R., Franssen F.F.J., de Vries E., Babesia bovis merozoites invade human, ovine, equine, porcine and caprine erythrocytes by a sialic acid-dependent mechanism followed by developmental arrest after a single round of cell fission, Int. J. Parasitol. (2003) 33:1595-1603.

[10] Gaur D., Mayer D.C.G., Miller L.H., Parasite ligand-receptor interactions during invasion of erythrocytes by Plasmodium merozoites, Int. J. Parasitol. (2004) 34:1413-1429.

[11] Gundula M.O., Gros P., Erythrocyte variants and the nature of their malaria protective effect, Cell. Microbiol. (2005) 7:753-763.

[12] Hadj-Kaddour H., Carcy B., Vallet A., Randazzo S., Delbecq S., Kleuskens J., et al., Recombinant protein $\mathrm{Bd} 37$ protected gerbils against heterologous challenges with isolates of Babesia divergens polymorphic for the $b d 37$ gene, Parasitology (2007) 134:187-196.

[13] Hudson B.G., Wegener L.J., Wingate J.M., Carraway K.L., Chemical studies of erythrocyte membrane glycoproteins from several species, Comp. Biochem. Physiol. (1975) 51B:127-135.

[14] Kania S.A., Allred D.R., Barbet A.F., Babesia bigemina: host factors affecting the invasion of erythrocytes, Exp. Parasitol. (1995) 80:76-84.

[15] Lewis D., Williams H., Infection of the Mongolian gerbil with the cattle piroplasm Babesia divergens, Nature (1979) 278:170-171.

[16] Lobo C.A., Babesia divergens and Plasmodium falciparum use common receptors, glycophorins A and $\mathrm{B}$, to invade the human red blood cell, Infect. Immun. (2005) 73:649-651.

[17] Malandrin L., L'Hostis M., Chauvin A., Isolation of Babesia divergens from carrier cattle blood using in vitro culture, Vet. Res. (2004) 35:1-9.

[18] Malandrin L., Marchand A.M., Chauvin A., Development of a microtitre-based spectrophotometric to monitor Babesia divergens growth in vitro, J. Microbiol. Methods (2004) 58:303-312.

[19] Pasvol G., How many pathways for invasion of the red blood cell by the malaria parasite? Trends Parasitol. (2003) 19:430-432. 
[20] Preiser P., Kaviratne M., Khan S., Bannister L., Jarra W., The apical organelles of malaria merozoites: host cell selection, invasion, host immunity and immune evasion, Microbes Infect. (2000) 2:1461-1477.

[21] Schuster F.L., Cultivation of Babesia and Babesia-like blood parasites: agents of an emerging zoonotic disease, Clin. Microbiol. Rev. (2002) 15:365-373.

[22] Vaskov B., Efremov G., Fourth haemoglobin type in sheep, Nature (1967) 216:593-594.

[23] Weatherall D.J., Miller L.H., Baruch D.I., Marsh K., Doumbo O.K., Casals-Pascual C., Roberts D.J., Malaria and the red cell, Hematology (2002) 35-57.

[24] Yokoyama N., Okamura M., Igarashi I., Erythrocyte invasion by Babesia parasites: current advances in the elucidation of the molecular interactions between the protozoan ligands and host receptors in the invasion stage, Vet. Parasitol. (2006) 138:22-32.

[25] Zhang W., Moreau E., Huang W., Chauvin A., Comparison of humoral response in sheep to Fasciola hepatica and $F$. gigantica experimental infection, Parasite (2004) 11:153-159.

[26] Zintl A., Westbrook C., Mulcahy G., Skerrett H.E., Gray J.S., Invasion, and short- and long-term survival of Babesia divergens (Phylum Apicomplexa) cultures in non-bovine sera and erythrocytes, Parasitology (2002) 124:583-588.

[27] Zintl A., Westbrook C., Skerrett H.E., Gray J.S., Mulcahy G., Chymotrypsin and neuraminidase treatments inhibit host cell invasion by Babesia divergens (Phylum Apicomplexa), Parasitology (2002) 125:45-50.

[28] Zintl A., Mulcahy G., Skerrett H.E., Taylor S.M., Gray J.S., Babesia divergens, a bovine blood parasite of veterinary and zoonotic importance, Clin. Microbiol. Rev. (2003) 16:622-636

[29] Zintl A., Skerrett H.E., Gray J.S., Brophy P.O., Mulcahy G., Babesia divergens (Phylum Apicomplexa) in vitro growth in the presence of calf serum, Vet. Parasitol. (2004) 122:127-130. 